\title{
Chemical elution treatment of synthetic diamond production wastewater contaminated soil
}

\author{
Guang-chen $\mathrm{SU}^{1,2,3}$, Xiang-xiang WANG ${ }^{1,2 *}$, Ying $\mathrm{YANG}^{1,2}$, Hai-shan ShI ${ }^{1,2,3}$ \\ ${ }^{1}$ College of Environmental and Energy Engineering, Anhui Jianzhu University, 230601 Hefei, China \\ ${ }^{2}$ Key Laboratory of Water Pollution Control and Wastewater Reuse, Anhui Jianzhu University, 230601 Hefei, China \\ ${ }^{3}$ Key Laboratory of Huizhou Architecture in Anhui Province, Anhui Jianzhu University, 230601 Hefei Anhui, China
}

\begin{abstract}
The optimal removal conditions of heavy metal Co and Ni composite contaminated soil were studied by the method of oscillating elution. The removal effects of different eluents on Co and Ni at different concentrations were discussed. The results showed that the removal efficiency of citric acid with the concentration of $0.2 \mathrm{~mol} \cdot \mathrm{L}^{-1}$ was the best, and the removal rates of $\mathrm{Co}$ and $\mathrm{Ni}$ were $73.40 \%$ and $73.03 \%$. On this basis, the effects of solid-liquid ratio, $\mathrm{pH}$ and elution time on the elution effect were investigated. The results show that the trend of the two heavy metals affected by the above three factors is about the same. As the solid-liquid ratio decreases, the elution effect gradually increases, but the growth range is different. As the $\mathrm{pH}$ increases, the elution effect of the two heavy metals decreases. In addition, the removal rates of the two heavy metals show a trend of rising first, then stabilizing, and then continuing to rise. Considering economic factors, time cost and treatment effect, the best elution conditions are solid-liquid ratio of 1: 20, $\mathrm{pH}$ value of 2 and elution time of $6 \mathrm{~h}$.
\end{abstract}

\section{Introduction}

Diamond is favored by today's society because of its unique physical and chemical properties, and is widely used in many fields such as industrial production and semiconductor research. However, natural diamonds are too expensive to be used for industrial production and scientific research. Therefore, because of the relatively low price, the artificial diamond is easy to obtain, and the supply channel is stable and loved by industrial enterprises. China's synthetic diamond production began in 1963. After more than 50 years of development, China's synthetic diamond production ranked first in the world ${ }^{[1]}$.

In the process of synthetic diamond production, a large amount of metal catalyst is consumed, and the main components of these metal catalysts are $\mathrm{Co}$ and $\mathrm{Ni}^{[2]}$. A large amount of waste liquid containing $\mathrm{Co}$ and $\mathrm{Ni}$ is produced in each cleaning stage of producing synthetic diamond. These waste liquids should have been treated to meet national standards and then discharged, but some enterprises have illegally discharged the waste liquids to reduce the operating costs, resulting in soil pollution ${ }^{[3]}$. Heavy metals will deteriorate the quality of soil environment after entering the soil, affecting crop growth, and may also enter the human body through the food chain. Human studies have shown that high concentrations of Co can inhibit plant growth and development ${ }^{[4]}$, excessive $\mathrm{Ni}$ causes changes in the structure and physiological processes of cells $\mathrm{s}^{[5]}$.

At present, the main methods for soil remediation of heavy metal pollution are physical, chemical and biological methods ${ }^{[6]}$. Among them, the soil elution method in chemical method is widely used because it can repair contaminated soil permanently, efficiently and economically ${ }^{[7]}$. In the chemical elution method, the choice of eluent is the key. Currently, the common types of eluents are artificial chelating agents, natural chelating agents, inorganic eluents and surfactants [8]. The representative of artificial chelating agent is ethylenediaminetetraacetic acid (EDTA), which is also the most widely used artificial chelating agent, and is suitable for most heavy metal pollution. The disadvantage is that it is not degradable and the reaction chelate is easily decomposed. Natural chelating agents mainly refer to small organic acids such as citric acid $\left(\mathrm{H}_{3} \mathrm{Cit}\right)$, which are natural organic eluents that have just begun to be used in recent years and are degradable under natural conditions. The most commonly used inorganic eluent is ferric chloride $\left(\mathrm{FeCl}_{3}\right)$, which has been shown to have a good removal effect on soil contaminated by $\mathrm{Cd}$ and $\mathrm{Pb}{ }^{[9]}$. Biosurfactants used in the removal of heavy metal pollution in soil are rhamnolipids, but their removal rate is low when used alone, and is generally used together with other eluents ${ }^{[10]}$.

In this paper, the wastewater containing $\mathrm{Co}$ and $\mathrm{Ni}$ was selected to contaminate the soil. Under different eluting agents and different concentrations of elution conditions, a stable and efficient eluent for removing Co

\footnotetext{
*Corresponding author: wangxiang156@126.com
} 
and Ni from soil was found. We will trying to explore the repair principle of $\mathrm{Co}$ and $\mathrm{Ni}$ contaminated soil through data analysis, in order to provide theoretical support for $\mathrm{Co}$ and $\mathrm{Ni}$ contaminated soil remediation.

\section{Materials and Methods}

\subsection{Trying to supply soil}

The experimental soil was collected from a synthetic diamond enterprise in Quanjiao County, Cangzhou City, Anhui Province, with a sampling depth of $20 \mathrm{~cm}$, one layer per $10 \mathrm{~cm}$, and layered sampling. After the soil sample is naturally air-dried, the bulky roots and stones and other debris are removed, and the ground is passed through a 100 mesh sieve for use. The soil heavy metal content was tested by Inductively Coupled Plasma Optical Emission Spectrometer (ICP-OES), and the digestion method used the national standard method to digest the soil. The basic properties of the soil are shown in Table 1.

Table 1. Sample soil basic properties

\begin{tabular}{|c|c|c|c|c|c|}
\hline $\mathrm{pH}$ & $\begin{array}{c}\text { Cation } \\
\text { exchange } \\
\text { capacity }\end{array}$ & $\begin{array}{c}\text { Organi } \\
\mathrm{c} \\
\text { matter }\end{array}$ & \multicolumn{3}{|c|}{$\begin{array}{c}\text { Heavy metal content } \\
/\left(\mathrm{mg} \mathrm{kg}^{-1}\right)\end{array}$} \\
\cline { 2 - 6 } & $\begin{array}{c}/\left(\mathrm{cmol} \mathrm{kg} \mathrm{kg}^{-1}\right. \\
\text { ) }\end{array}$ & $/ \%$ & $\mathrm{Co}$ & $\mathrm{Ni}$ & $\mathrm{Cu}$ \\
\hline 6.18 & 70 & 5.36 & 200.54 & $\begin{array}{c}3735.4 \\
1\end{array}$ & 11868.75 \\
\hline
\end{tabular}

\subsection{Experimental materials and equipment}

The main experimental reagents are: acetic $\operatorname{acid}\left(\mathrm{CH}_{3} \mathrm{COOH}\right)$, propionic $\operatorname{acid}\left(\mathrm{CH}_{3} \mathrm{CH}_{2} \mathrm{COOH}\right)$, n-butyric acid $\left(\mathrm{C}_{4} \mathrm{H}_{8} \mathrm{O}_{2}\right)$, citric acid $\left(\mathrm{H}_{3} \mathrm{Cit}\right)$, tartaric acid $\left(\mathrm{C}_{4} \mathrm{H}_{6} \mathrm{O}_{6}\right)$, oxalic $\operatorname{acid}\left(\mathrm{H}_{2} \mathrm{C}_{2} \mathrm{O}_{4}\right)$, disodium edetate(EDTA-2Na), hydrochloric acid( $\mathrm{HCl})$, sodium hydroxide $(\mathrm{NaOH})$, nitric $\operatorname{acid}\left(\mathrm{HNO}_{3}\right)$, hydrofluoric $\operatorname{acid}(\mathrm{HF})$, perchloric $\operatorname{acid}\left(\mathrm{HClO}_{4}\right)$. The reagents were of analytical grade and purchased from Sinopharm Chemical Reagent Co, Ltd. The experimental water was ultrapure water.

Main equipment of experiment: constant temperature oscillator, centrifuge, ICP-OES, microwave digestion instrument, ultra pure water machine.

\subsection{Experimental methods}

We weighed $1.0 \mathrm{~g}$ of soil sample into a $50 \mathrm{~mL}$ polyethylene centrifuge tube and added the eluent according to the different experimental methods described below. The tube was placed in a constant temperature oscillator at $25^{\circ} \mathrm{C}$ under room temperature with a rotation speed of $150 \mathrm{r} \bullet \mathrm{min}^{-1}$. After the shaking, the tube was taken out and centrifuged at $3000 \mathrm{r} \bullet \mathrm{min}^{-1}$ for $15 \mathrm{~min}$ in a high-speed centrifuge. The supernatant was then passed through a $0.45 \mu \mathrm{m}$ microporous membrane to a $10 \mathrm{~mL}$ centrifuge tube for testing. A blank experiment was also performed at the same time Each set of experiments was repeated 3 times.

\subsubsection{Eluent screening}

The natural organic eluent is selected from the group consisting of citric acid, tartaric acid and oxalic acid. The concentration gradient is $0.05,0.10,0.20,0.30,0.50$, $0.70,1.00 \mathrm{~mol} \cdot \mathrm{L}^{-1}$; the artificial chelating agent is disodium edetate, the concentration gradient is $0.01,0.02$, $0.04,0.08,0.10,0.20,0.30 \mathrm{~mol} \cdot \mathrm{L}^{-1}$; small molecule organic eluent used acetic acid, propionic acid, n-butyric acid, concentration gradients of $0.05,0.10,0.20,0.30$, $0.50,0.70,1.00 \mathrm{~mol} \cdot \mathrm{L}^{-1}$. The experimental conditions were initially determined to be a solid-liquid ratio of 1:20 and a shaking time of $24 \mathrm{~h}$.

\subsubsection{Screening of eluent conditions}

Eluent time: Select the best acid and its optimum concentration for $\mathrm{Co}$ and $\mathrm{Ni}$ removal. Experiment with this concentration. The solid-liquid ratio is $1: 20$, and the oscillation time is $0,0.5,1,3,6,12$, respectively. $24 \mathrm{~h}$.

Solid-liquid ratio: The best acid and its optimum concentration for $\mathrm{Co}$ and $\mathrm{Ni}$ removal were selected. The experiment was carried out according to this concentration. The shaking time was $24 \mathrm{~h}$, and the solid-liquid ratio was $1: 2,1: 5,1: 10$, respectively. $1: 15: 1: 20,1: 30,1: 40$.

$\mathrm{pH}$ : The best acid and its optimum concentration for $\mathrm{Co}$ and $\mathrm{Ni}$ removal were selected. The experiment was carried out according to this concentration. The solid-liquid ratio was 1:20, the shaking time was $24 \mathrm{~h}$, and the $\mathrm{pH}$ was adjusted to 2 with hydrochloric acid and sodium hydroxide $3,4,5,6,7,8,9,10$.

\subsection{Data processing}

The data processing was completed under EXCEL2010, and the image was drawn using OrijinPro2017. The removal rate is the ratio of the heavy metal content in the eluent to the total heavy metal content in the soil.

\section{Results and analysis}

$\mathrm{Co}$ and $\mathrm{Ni}$ are the main elements of the contaminated soil of synthetic diamond production wastewater. The measured values of $\mathrm{Co}$ and $\mathrm{Ni}$ in the trial soil are the standard values specified in the "Soil and Water Quality Risk Management and Control Standards (Trial) " 10 times and 25 times of the class, which is heavily polluted, poses a serious threat to human and animal and plant growth and development.

\subsection{Eluent screening}

Fig. 1 (I) shows the effect of different concentrations of organic acids on the removal rate of Co. Fig. 1 (II) shows the effect of different concentrations of organic acids on the removal rate of $\mathrm{Ni}$. 


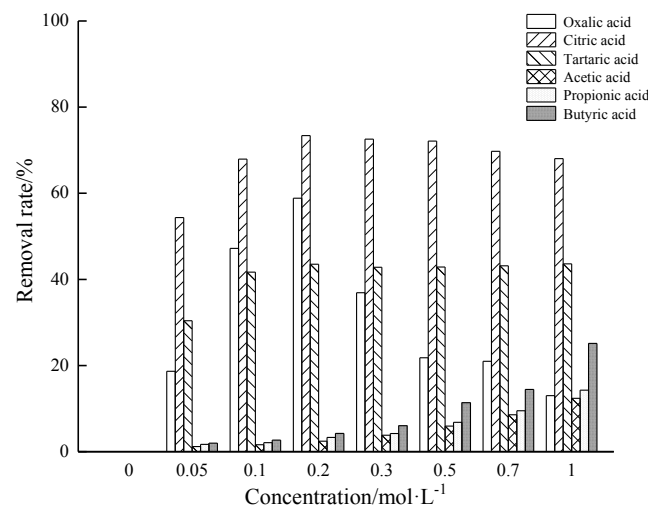

(I)

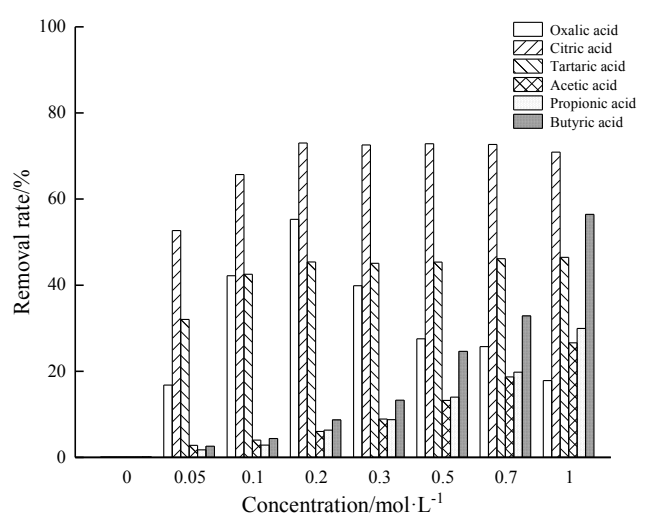

(II)

Fig.1. Effect of different concentrations of organic acids on the removal rates of heavy metals $\mathrm{Co}(\mathrm{I})$ and $\mathrm{Ni}$ (II)

It can be seen from Fig. 1 that the small molecular organic acid performs poorly on both metal removal rates compared with the natural organic acid, but the removal efficiency of n-butyric acid to nickel at high concentration $\left(1.00 \mathrm{~mol} \cdot \mathrm{L}^{-1}\right)$ can reach $56.45 \%$. The removal rate of oxalic acid to two heavy metals was good at $0.2 \mathrm{~mol} \cdot \mathrm{L}^{-1}$, which could reach $58.86 \%$ and $55.30 \%$. However, the removal rate decreased with the further increase of concentration. When the concentration of citric acid is $0.2 \mathrm{~mol} \cdot \mathrm{L}^{-1}$, the removal rate of heavy metals of $\mathrm{Co}$ and $\mathrm{Ni}$ reaches the maximum screening value of $73.40 \%$ and $73.03 \%$. When the concentration of tartaric acid is $0.05 \mathrm{~mol} \cdot \mathrm{L}-1$, the removal rates of heavy metals of $\mathrm{Co}$ and $\mathrm{Ni}$ are $30.39 \%$ and $32.04 \%$. When the concentration is increased to $0.20 \mathrm{~mol} \cdot \mathrm{L}^{-1}$. The removal rates of tartaric acid to the two heavy metals were $43.53 \%$ and $45.38 \%$, respectively. With the further increase of concentration, the removal effect of tartaric acid on the two heavy metals began to balance, and the removal rates of the two heavy metals were maintained at about $43 \%$. The removal efficiency of citric acid on Co and $\mathrm{Ni}$ is the best at each concentration. When the concentration reaches $0.20 \mathrm{~mol} \cdot \mathrm{L}^{-1}$, the removal rate of the two heavy metals is optimal, and increasing the concentration will reduce the removal rate. Compared with small molecule organic acids, natural organic acids show better removal effects, mainly because natural organic acids mainly increase the mobility of metal ions by complexing with metal ions to form stable metal complexes. It promotes the precipitation of heavy metals from the soil surface and can inhibit the re-adsorption of heavy metals, thereby achieving the effect of heavy metal removal ${ }^{[11]}$.Studies have shown that the amount of hydroxyl and carboxyl groups in organic acids affects the removal rate. Citric acid is structurally similar to tartaric acid, the former being dihydroxytricarboxylic acid and the latter being dihydroxydicarboxylic acid. In the case of the same amount of substance, citric acid has more carboxyl groups than tartaric acid, so the removal of citric acid is superior to tartaric acid ${ }^{[12]}$.From the removal rate, when the concentration of tartaric acid was 0.20 $\mathrm{mol} \cdot \mathrm{L}^{-1}$, the removal rates of the two heavy metals were $43.53 \%$ and $45.38 \%$, while at the same concentration the removal rates of citric acid to the two heavy metals were $73.40 \%$ and $73.03 \%$.With the change of concentration, the removal rate of citric acid on the two heavy metals first increased and then decreased. This is because when the concentration of citric acid is not very high, $\mathrm{H}^{+}$can be consumed in a large amount. And the concentrations of $\mathrm{H}_{2} \mathrm{Cit}^{-}, \mathrm{HCit}^{2-}$ and $\mathrm{Cit}^{3-}$ are sufficient to generate a large amount of metal complexes, and when the concentration of citric acid is too high, $\mathrm{H}^{+}$cannot be significantly consumed, and the remaining $\mathrm{H}^{+}$inhibits the ionization of $\mathrm{H}_{2} \mathrm{Cit}^{-}, \mathrm{HCit}^{2-}$ and $\mathrm{Cit}^{3-}$, thereby inhibiting the complexation reaction. The occurrence of this leads to a decrease in the removal rate.

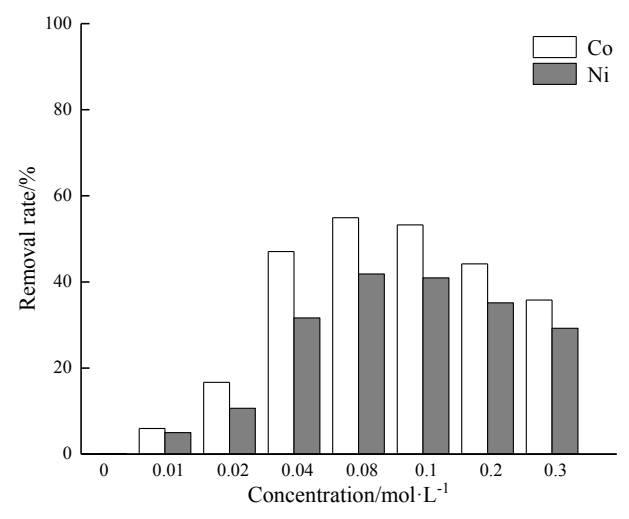

Fig. 2. Effect of different concentrations of EDTA-2Na on the removal rates of heavy metals $\mathrm{Co}$ and $\mathrm{Ni}$

EDTA has been widely used as a common artificial chelating agent for the removal of heavy metals, but the solubility of EDTA ( $0.02 \mathrm{~g} / 100 \mathrm{ml})$ is insufficient to meet the needs of the experiment, so the solubility of EDTA-2Na is higher $(11.1 \mathrm{~g} / 100 \mathrm{ml})$. It can be seen from Fig. 2 that when the concentration of EDTA-2Na is about $0.08 \mathrm{~mol} \cdot \mathrm{L}-1$, the removal rates of heavy metals $\mathrm{Co}$ and $\mathrm{Ni}$ are the best, reaching $54.91 \%$ and $41.84 \%$. It can be seen that EDTA-2Na has the optimal concentration for the removal of the two heavy metals, but the removal rate at the optimal concentration is still less than the average removal rate of citric acid. Although EDTA-2Na has a high solubility, it still has a large gap compared with natural organic acids such as citric acid. In addition, since the former is expensive, not 
easy to degrade, and the reaction product is unstable, EDTA-2Na is not considered as a separate Soil eluent ${ }^{[13]}$. Instead, citric acid is a soil eluent that is more easily degradable and has less environmental impact. Comprehensive efficiency and economic considerations, so the optimal eluent for the specific soil of this experiment is citric acid, the optimal concentration is 0.2 $\mathrm{mol} \cdot \mathrm{L}^{-1}$.

\subsection{Screening of eluent conditions}

Organic acids can be affected by many factors in the process of elution heavy metals in soil. The elution time, solid-liquid ratio and $\mathrm{pH}$ value of the eluent are the main factors affecting the elution effect.

\subsubsection{Effect of eluent time on elution effect}



Fig. 3. Effect of time on removal rate of heavy metals Co and $\mathrm{Ni}$

Time is one of the important factors affecting the elution effect. To achieve a higher removal rate, it is necessary to maintain sufficient elution time ${ }^{[14]}$. It can be seen from Fig. 3 that the removal rate of the two heavy metals changes with the same trend with time, and the removal effect of metallic $\mathrm{Ni}$ is slightly higher than that of metallic Co. When the elution time was $1 \mathrm{~h}$, the removal rates of $\mathrm{Co}$ and $\mathrm{Ni}$ were $11.16 \%$ and $12.13 \%$. When the elution time reached 6 hours, the removal rates of the two heavy metals could reach $45.43 \%$ and $43.59 \%$. When the elution time is further increased to $12 \mathrm{~h}$, the removal rate of the two heavy metals is less than $20 \%$, which can reach $56.48 \%$ and $55.11 \%$. After that, the elution effect enters the stationary phase. At the end of 24 $\mathrm{h}$ of elution, the removal rates of heavy metals were $73.40 \%$ and $73.03 \%$. It is worth noting that at time 0 , the removal rates of the two heavy metals are still $4.38 \%$ and $6.06 \%$, which also explains that the removal rate of the two heavy metals by citric acid increases significantly during the initial stage of leaching. The removal rate increased slowly during the $12 \mathrm{~h}$ to $24 \mathrm{~h}$ period. This was because the citric acid mixed with the soil preferentially complexed with the acid extractable state in the soil surface components, and the removal rate increased rapidly. However, as the acid extractable state decreases, the reaction rate gradually decreases, followed by a plateau. This is similar to the study of heavy metals in citric acid remove e-waste contaminated soil ${ }^{[15]}$. When the elution time continues to increase, the soil particles begin to dissolve, other forms of heavy metals inside the granules begin to separate out, and complexation with citric acid occurs, and the removal rate is increased again. However, compared to the acid extractable state in the soil, other forms of heavy metals can be less translated with citric acid, so the rate of growth is slower. It is expected that as the elution time is further increased, the complexation reaction tends to be saturated, and the removal rate will also tend to be stable. The elution time can be chosen for $6 \mathrm{~h}$, taking into account the time cost and removal effect.

\subsubsection{Effect of solid-liquid ratio on elution effect}

Fig. 4 is a graph showing the change in the removal rate of citric acid to heavy metal $\mathrm{Co}$ and $\mathrm{Ni}$ as the solid to liquid ratio is lowered. As shown in the figure, the removal curves of citric acid for the two heavy metals are basically the same, which are slightly fluctuated with the decrease of the solid-liquid ratio, but generally show an upward trend. When the ratio of solid to liquid is higher, the removal efficiency of citric acid on both heavy metals is not good. The removal rates of the two heavy metals in the case of solid-liquid ratio $1: 2$ are $7.32 \%$ and $8.02 \%$. The liquid ratio was reduced to $1: 5$, and the removal rates of $\mathrm{Co}$ and $\mathrm{Ni}$ were increased to $27.76 \%$ and $26.59 \%$. When the solid-liquid ratio was $1: 10$ and $1: 15$, the removal rate of heavy metals was not improved, and remained at about $45 \%$. When the solid-liquid ratio is $1: 20$, the removal rate of the two heavy metals can reach $73.40 \%, 73.03 \%$; and when the solid-liquid ratio is reduced to $1: 30$, even $1: 40$, the removal effect of the two heavy metals changes, the growth is not obvious. When the solid-liquid ratio is $1: 40$, the removal rate is only about 7\% higher than 1:20. It can be seen from Fig. 4 that the removal rate shows a stepwise rise with the decrease of the solid-liquid ratio, which may be due to the complexation reaction of citric acid with heavy metals, and the amount of heavy metals is much higher than the amount of acid. In the case of increasing the acid content, the reaction proceeds. In the process of increasing the proportion of acid, the area of the eluent that can be contacted by the unit mass of soil particles reaches the limit, the reaction rate is slowed down, and $\mathrm{H}^{+}$begins to inhibit the reaction, which is the same as the former ${ }^{[16]}$. Therefore, in the case of continuing to reduce the solid-liquid ratio without a large increase, considering the elution cost, it is recommended to use 1:20 as the optimal eluent solid-liquid ratio. 


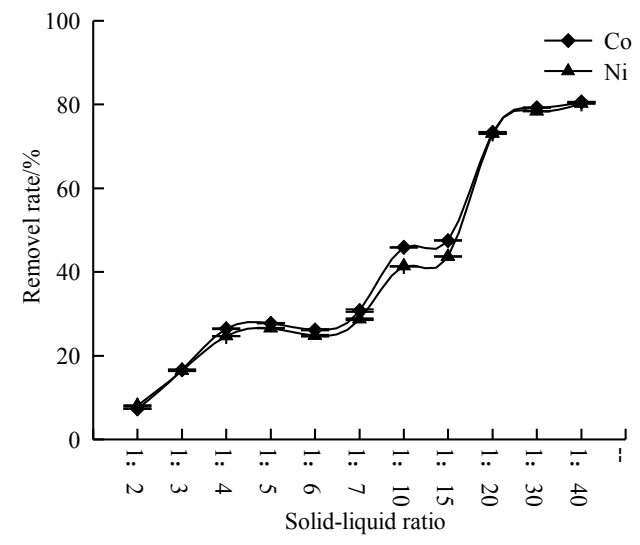

Fig. 4. Effect of solid-liquid ratio on removal rate of heavy metals $\mathrm{Co}$ and $\mathrm{Ni}$

\subsubsection{Effect of $\mathrm{pH}$ on the elution effect}

The $\mathrm{pH}$ plays a crucial role in the formation of the complexation reaction. The concentration of $\mathrm{H}^{+}$affects the number of ligands that can complex with heavy metal ions ${ }^{[17]}$. It can be seen from Fig. 5 that the removal rates of heavy metals $\mathrm{Co}$ and $\mathrm{Ni}$ are the same as those affected by $\mathrm{pH}$. The removal effect is best when the $\mathrm{pH}$ is low, and the closer the $\mathrm{pH}$ is 7 , the more obvious the treatment effect is decreased. In the alkaline environment, the treatment effect of citric acid on the two heavy metals is not obvious, which is the same as the previous research results ${ }^{[18]}$. When citric acid enters the soil, it releases its own $\mathrm{H}^{+}$, competes with the heavy metals in the soil, seizes the adsorption sites, and promotes the desorption of soil particles. The more the number of $\mathrm{H}^{+}$entering the soil, the more obvious the leaching effect of heavy metals in the soil, and the leaching efficiency is proportional to the acidity ${ }^{[19]}$. When the $\mathrm{pH}$ was 2 , the elution effect was the best. The removal rates of $\mathrm{Co}$ and $\mathrm{Ni}$ were $79.92 \%$ and $79.24 \%$. When the $\mathrm{pH}$ of citric acid is 7 , the removal rates of the two heavy metals are $0.38 \%$ and $3.50 \%$. Under alkaline conditions, Co can hardly be removed, and only a small amount of $\mathrm{Ni}$ is eluted. Therefore, it is recommended to use citric acid with $\mathrm{pH} 2$ for elution.

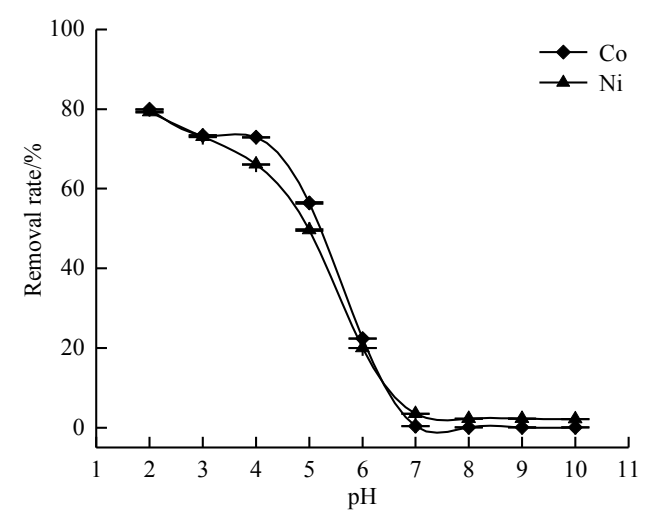

Fig. 5. Effect of $\mathrm{pH}$ on removal rate of heavy metals $\mathrm{Co}$ and $\mathrm{Ni}$

\section{Discuss}

As an environmentally friendly complexing agent, citric acid is widely used in heavy metal removal and heavy metal contaminated soil remediation research. Amjad H. El-Sheikh et al. used citric acid to remove zinc and Co contamination in water ${ }^{[20]}$. Zhang Jia et al. used citric acid to treat soil chromium pollution in chromium residue dumping sites. The removal rate of chromium can reach $80.70 \%{ }^{[21]}$. Min $\mathrm{Yu}$ et al used citric acid as the elution solution to recover Co and lithium in the waste lithium battery. The elution time was 70 minutes, the solid-liquid ratio was 1:25, and the elution rate was $99 \%$ when the reaction temperature was $70{ }^{\circ} \mathrm{C}{ }^{[22]}$. Studies have shown that the use of citric acid as a elution agent to remove Co and Ni from soil is a feasible method. The physicochemical properties of the soil after citric acid treatment will change slightly, and the total organic carbon will increase slightly, which may be related to the residual citric acid in the soil. At the same time, the treated soil also has problems such as $\mathrm{pH}$ rise and soil compaction, but the residue of citric acid in the soil can be biodegraded compared with other eluents, so that the influence on the soil can be gradually eliminated.

\section{Conclusion}

Compared with other eluents, citric acid has the best effect on the removal of $\mathrm{Co}$ and $\mathrm{Ni}$, and citric acid as a new environmentally friendly eluent has little effect on the physical and chemical properties of the soil. After the citric acid enters the soil, the hydroxyl group and carboxyl group in the acid root begin to combine with the heavy metal to form a stable metal complex, which further promotes soil desorption. However, when heavy metal adsorption reaches saturation, excessive citrate inhibits the formation of metal complexes. Therefore, citric acid with an ion concentration of $0.2 \mathrm{~mol} \cdot \mathrm{L}^{-1}$ was selected as the optimal eluent.

In the study of factors affecting the elution effect, such as elution time, solid-liquid ratio and $\mathrm{pH}$, the elution time was $6 \mathrm{~h}$, the solid-liquid ratio was 1:20, and the solution $\mathrm{pH}=2$ was the best elution condition.

$0.2 \mathrm{~mol} \cdot \mathrm{L}^{-1}$ citric acid eluent was used, the ratio of solid to liquid was $1: 20, \mathrm{pH}=2$, and the elution time was $6 \mathrm{~h}$. The removal rates of $\mathrm{Co}$ and $\mathrm{Ni}$ heavy metals can reach $53.26 \%$ and $58.49 \%$.

\section{References}

1 Luo Xiyu, Xu Yanjun, LiuYibo. The development and key technological improvement of synthetic diamond in China [J]. Powder Metall Industry. 2016, 26(1):1-13.

2 Nojkina A V, Laptev A I, Ermolaev A A. Influence of Synthesis and Composition conditions on Strength Characteristics of Synthetic Carbonado-Type Diamonds[J]. High Pressure Res., 2002, 22(3-4):545-549.

3 Hao Xuekui, Li Xiaohong, Wang Sanfan. 
TREATMENT OF SYNTHETIC DIAMOND WASTEWATER [J]. Technol. Water Treat., 2007, 33(10):55-57.

4 Xie Hongke, Zou Chaohui,Peng Xuanming. Research Progress on Remediation of Heavy Metal Polluted Soil by Co [J]. XianDai NongYe KeJi, 2013(7):222-223.

5 Wang Rui, Yu Zhongling, Guang Yang. Overview on Phytoremediation of $\mathrm{Ni}$ Contaminated Soil [J]. Environ. Sci. Manage., 2013, 38(8):111-114.

6 ao Z. Review on Remediation Technologies of Soil Contaminated by Heavy Metals[J]. Procedia Environ. Sci., 2012, 16(4):722-729.

7 Yang X, Mao X, Shao X, et al. Enhanced Techniques of Soil Washing for the Remediation of Heavy Metal-Contaminated Soils[J]. Agric. Res., 2018.

8 Cuan Limin, Zhao Tongke, Zheng Huaiguo8. Research Advances in Remediation of Heavy Metal Contaminated Soils [J]. Environ. Sci. Technol., 2014.37(S2):213-222.

9 Makino T, Takano H, Kamiya T, et al. Restoration of cadmium-contaminated paddy soils by washing with ferric chloride: $\mathrm{Cd}$ extraction mechanism and bench-scale verification[J]. Chemosphere, 2008, 70(6):0-1043.

10 Feng Jing,Zhang Zengqiang, Li Nian. Washing of heavy metal contaminated soil around a lead-zinc smelter by several chelating agents and the leached soil utilization [J]. HGXUBP, 2015, 9(11).

11 Xie Dan, Xu Renkou,Jiang Xin. Effect of Organic Acids on Competitive Adsorption of $\mathrm{Cu}(\mathrm{II}), \mathrm{Pb}$ ( II ) and Cd( II ) by Variable Charge Soils [J]. J. AgroEnviron. Sci., 2006, 25(3).

12 Shaker M A, Albishri H M. Dynamics and thermodynamics of toxic metals adsorption onto soil-extracted humic acid[J]. Chemosphere, 2014, 111:587-595.

13 Beiyuan J, Tsang D C W, Valix M, et al. Combined application of EDDS and EDTA for removal of potentially toxic elements under multiple soil washing schemes $[\mathrm{J}]$. Chemosphere, 2018:

\section{S0045653518307306.}

14 Sun Tao, Lu Kouping, Wang Hailong. Advance in washing technology for remediation of heavy metal contaminated soils: effects of eluants and washing conditions [J]. J. Zhejiang A\&F Univ., 2015, 32(1):140-149.

15 Xing Yu, Dang Zhi, Sun Beili. Removal of Heavy Metals from Electronic Waste Contaminated Soil by Washing with Citric Acid [J]. Environ. Prot. Chem. Ind., 2014, 34(2):110-113.

16 Yi Longsheng, Wang Wenyan, Tao Ye. Removing Heavy Metals in Contaminated Soils by the Organic Acids [J]. J. Agro- Environ. Sci., 2013(4):701-707.

17 Wang Jianbing, Chen Zhipeng, Duan Mingxing. Leaching characteristics and release law of heavy metals from the mining mullock and tailings in nonferrous metal mining industry [J]. Ecol. Environ. Sci., 2014(2).

18 Xiazhadan Abulikimu, Aikebaier Yilahong, Tursun Tuerhong. Effect of $\mathrm{pH}$ on the Complex Reaction of Humic acid, Fulvic Acid with Cd2+ [J]. Xinjiang Agric. Sci., 2012, 49(9):1701-1706.

19 Chen Xinyuan, Wu Yangqing. Remediation mechanism of multi-heavy metal contaminated soil by usingdifferent chemical washing agents[J]. Chin. J. Environ. Eng., 2018, 12(10):135-144.

20 Amjad H.El-Sheikh ,Ismail I.Fasfous, Rawan M.Al-Salamin, et al. Immobilization of citric acid and magnetite on sawdust for competitive adsorption and extraction of metal ions from environmental waters[J]. J. Environ. Chem. Eng., 2018,6(4):5186-5195

21 Zhang Jia, Chen Honghan, Zhang Yankun,. Experimental study on the performance of chromium removal from soil by citric acid leaching [J]. Acta Sci. Circ., 2015, 35(7):2247-2253.

22 Min Yu,Zehui Zhang,Feng Xue.A more simple and efficient process for recovery of Co and lithium from spent lithium-ion batteries with citric acid[J]. Sep. Puri. Technol.,215(2019)398-402. 